\title{
Gastric Hyperplastic Polyps after Argon Plasma Coagulation for Gastric Antral Vascular Ectasia in Patients with Liver Cirrhosis: A Case Suggesting the "Gastrin Link Theory"
}

\begin{abstract}
Ken Nishino ${ }^{1}$, Miwa Kawanaka ${ }^{1}$, Mitsuhiko Suehiro ${ }^{1}$, Naoko Yoshioka ${ }^{2}$, Jun Nakamura ${ }^{3}$, Noriyo Urata ${ }^{1}$, Tomohiro Tanigawa ${ }^{1}$, Takako Sasai ${ }^{1}$, Takahito Oka ${ }^{1}$, Yasumasa Monobe ${ }^{4}$, Yoshiaki Saji ${ }^{5}$, Hirofumi Kawamoto ${ }^{1}$ and Ken Haruma ${ }^{1}$
\end{abstract}

\begin{abstract}
:
We herein report a case of gastric hyperplastic polyps after argon plasma coagulation (APC) for gastric antral vascular ectasia (GAVE) in the antrum of a 65-year-old man with liver cirrhosis and hypergastrinemia induced by long-term proton pump inhibitor (PPI) use. Two years after APC therapy, endoscopy demonstrated multiple gastric polyps in the antrum and angle. A gastric polyp biopsy indicated foveolar epithelium hyperplasia, which was diagnosed as gastric hyperplastic polyps. One year after switching to an $\mathrm{H} 2$ blocker antagonist, endoscopy revealed that the polyps and GAVE had disappeared, with normal gastrin levels suggesting that PPI-induced hypergastrinemia had caused gastric hyperplastic polyps after APC therapy, and the polyps had disappeared after discontinuing PPIs.
\end{abstract}

Key words: gastric polyp, hypergastrinemia, argon plasma coagulation, proton pump inhibitor, gastric antral vascular ectasia

(Intern Med 60: 1019-1025, 2021)

(DOI: 10.2169/internalmedicine.5837-20)

\section{Introduction}

Esophageal and gastric varices, portal hypertensive gastropathy, and gastric antral vascular ectasia (GAVE) are common and characteristic findings observed by endoscopic examinations in patients with liver cirrhosis (1-6). They are the cause of anemia and acute or chronic gastrointestinal bleeding in patients with liver cirrhosis (1-6).

Although GAVE was first described in 1953 by Rider et al. as a cause of massive gastric bleeding (7), its etiology is not fully understood. Treatment includes conservative measures, such as acid suppression agents, blood transfusion, and endoscopic therapy. Endoscopic therapy, especially coagulation with argon plasma coagulation (APC), has become in- creasingly popular for the treatment of GAVE (8-10). In general, complications of APC for GAVE such as perforation and bleeding, are rare because of the superficial coagulation effect (11-13). However, rare cases of gastric polyps developing after APC therapy for GAVE have been reported (14-20) and termed "portal hypertension-associated polyps" or "portal hypertensive polyps" (21-24). The pathogenesis of gastric hyperplastic polyps is still unknown, but it is thought that the exaggerated repair of mucosal damage $(25,26)$ or hypergastrinemia may play a role in the development of the polyps (27-29).

We herein report a rare case of gastric hyperplastic polyps following APC of GAVE. In this case, hypergastrinemia was caused by the prolonged use of a proton pump inhibitor (PPI) at the time of the diagnosis of gastric hyperplastic pol-

\footnotetext{
${ }^{1}$ Department of General Internal Medicine 2, Kawasaki Medical School General Medical Center, Japan, ${ }^{2}$ Department of Hepatology and Pancreatology, Kawasaki Medical School, Japan, ${ }^{3}$ Department of Clinical Pathology and Laboratory, Kawasaki Medical School, Japan, ${ }^{4}$ Department of Pathology, Kawasaki Medical School General Medical Center, Japan and ${ }^{5}$ Department of Surgery, Ishikawa Hospital, Japan Received: July 11, 2020; Accepted: September 7, 2020; Advance Publication by J-STAGE: October 28, 2020 Correspondence to Dr. Miwa Kawanaka, m.kawanaka@med.kawasaki-m.ac.jp
} 


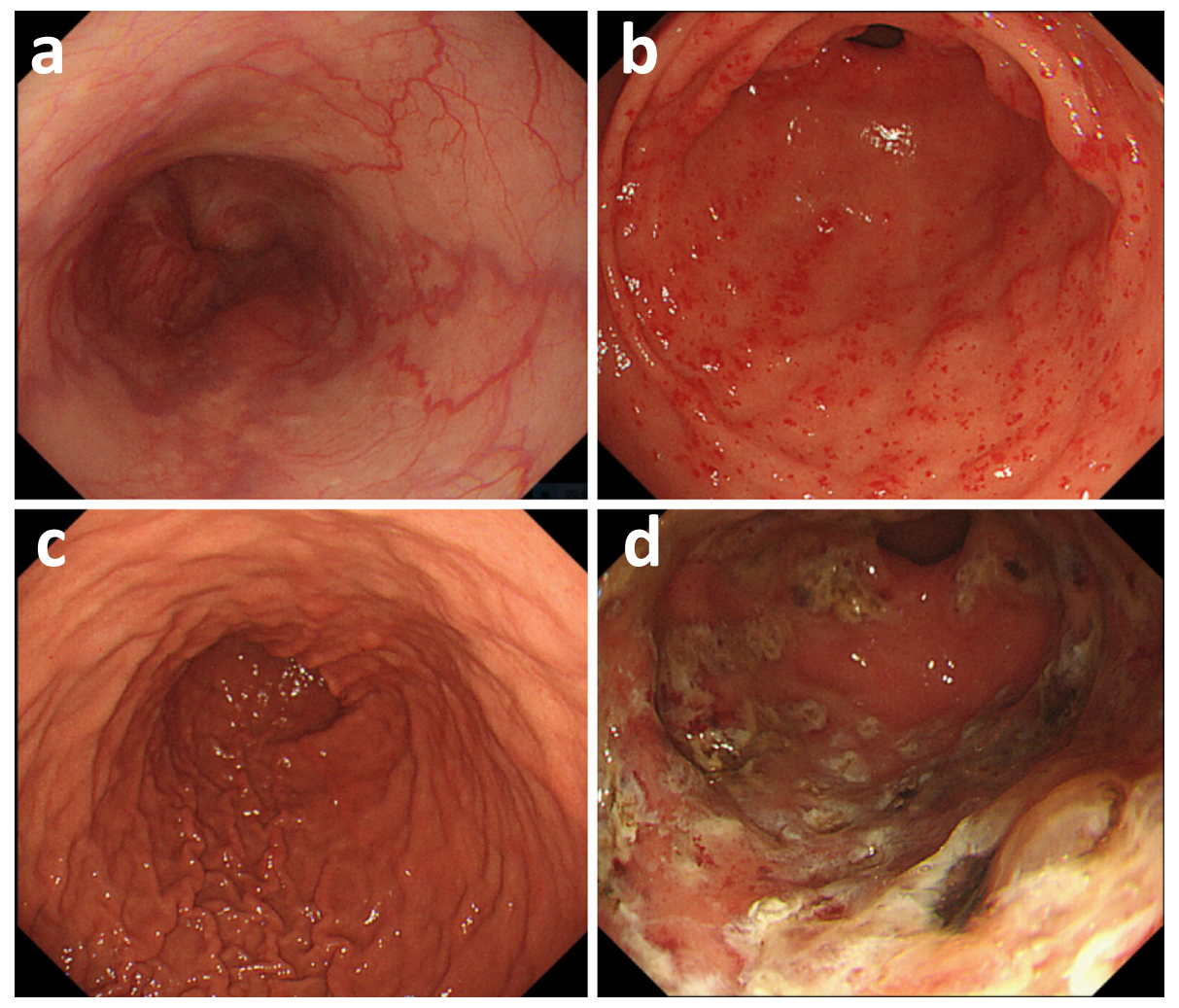

Figure 1. Initial endoscopic findings. Endoscopy revealed mild esophageal varix in the lower esophagus (a) and multiple red spots in the antrum with multiple low polyploid lesions (b). No atrophy or polyps were present (c). Multiple erosions after APC therapy (d). APC: argon plasma coagulation

yps. The discontinuance of PPIs and change to an H2receptor antagonist with rebamipide normalized the level of serum gastrin and promoted the natural disappearance of gastric polyps with a good prognosis of GAVE.

\section{Case Report}

A 65-year-old man with liver cirrhosis and portal hypertension associated with hepatitis $\mathrm{C}$ virus was referred to us from an outside facility for the further evaluation of refractory iron deficiency anemia $(\mathrm{Hb}, 9.1 \mathrm{~g} / \mathrm{mL})$. He had a history of long-term use of PPIs because of suspicion of gastrointestinal bleeding.

Initial upper gastrointestinal endoscopy revealed mild esophageal varix in the lower esophagus and multiple red spots in the antrum with multiple low polyploid lesions, which were diagnosed as GAVE associated with portal hypertension and raised type-erosive gastritis. No atrophy and no polyps were found in the stomach, and APC therapy was performed for GAVE (Fig. 1). After APC therapy, a PPI (esomeprazole $20 \mathrm{mg} /$ day) was subsequently readministered. Repeated endoscopies (second and third) showed multiple ulcers in the antrum at one week after APC (Fig. 2a, b), as well as multiple scars and reddish small polypoid lesions in the antrum at two months after APC (Fig. 2c, d). PPI treatment was continued for the patient, and the clinical course was good except for moderate iron deficiency anemia $(\mathrm{Hb}$, $11.0 \mathrm{~g} / \mathrm{mL}$ ).
Five months after APC therapy, he was diagnosed with hepatocellular carcinoma (HCC) $($ size $1 \times 1 \mathrm{~cm})$ and treated with radiofrequency ablation without recurrence. Eight months later, he was treated for hepatitis $\mathrm{C}$ using direct acting antivirals and went into remission. Two years and six months after APC therapy, a fourth endoscopy demonstrated multiple reddish polypoid lesions in the anterior of the antrum and greater curvature of the stomach (Fig. 3). Biopsy specimens from gastric polyps in the antrum and angle indicated hyperplasia of the foveolar epithelium with edema and capillary dilation (Fig. 4), and the lesions were diagnosed as gastric hyperplastic polyps. The level of fasting serum gastrin was $817 \mathrm{pg} / \mathrm{mL}$ (normal range: $50-150 \mathrm{pg} / \mathrm{mL}$ ), and serum Helicobacter pylori antibody was negative on the day of endoscopy. Therefore, we considered the cause of gastric hyperplastic polyps to be hypergastrinemia induced by PPIs and switched from a PPI to an H2 blocker antagonist (famotidine $40 \mathrm{mg} /$ day) and a mucoprotective agent (rebamipide $300 \mathrm{mg} /$ day).

One year later, a fifth endoscopy revealed that all polyps had completely disappeared, and GAVE was not present (Fig. 5). The fasting level of serum gastrin was in the normal range $(121 \mathrm{pg} / \mathrm{mL})$. In addition, a complete improvement in iron deficiency anemia was found $(\mathrm{Hb}, 15.4 \mathrm{~g} / \mathrm{mL})$. Four and five years after APC therapy, the sixth and seventh endoscopic examinations were performed, respectively, and no polyps or GAVE were observed. The clinical course of liver cirrhosis was stable during the follow-up period 



Figure 2. Repeated endoscopies (second and third). Multiple ulcers in the antrum at one week after APC (a, b) and multiple scars and reddish small polypoid lesions in the antrum at two months after APC (c, d). APC: argon plasma coagulation

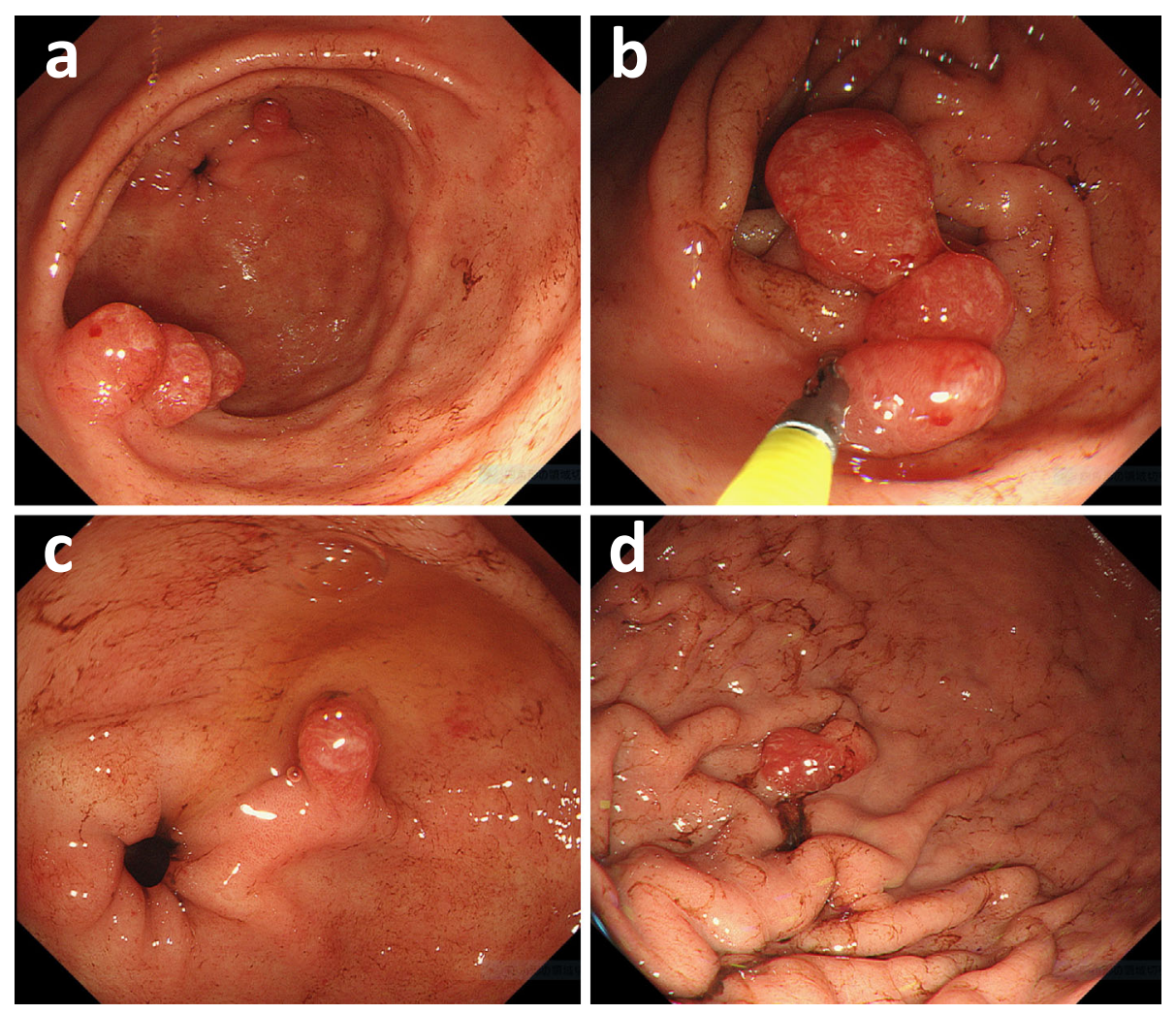

Figure 3. Two years after APC therapy, a fourth endoscopy demonstrated multiple reddish polypoid lesions in the anterior of the antrum and greater curvature of the stomach. APC: argon plasma coagulation 

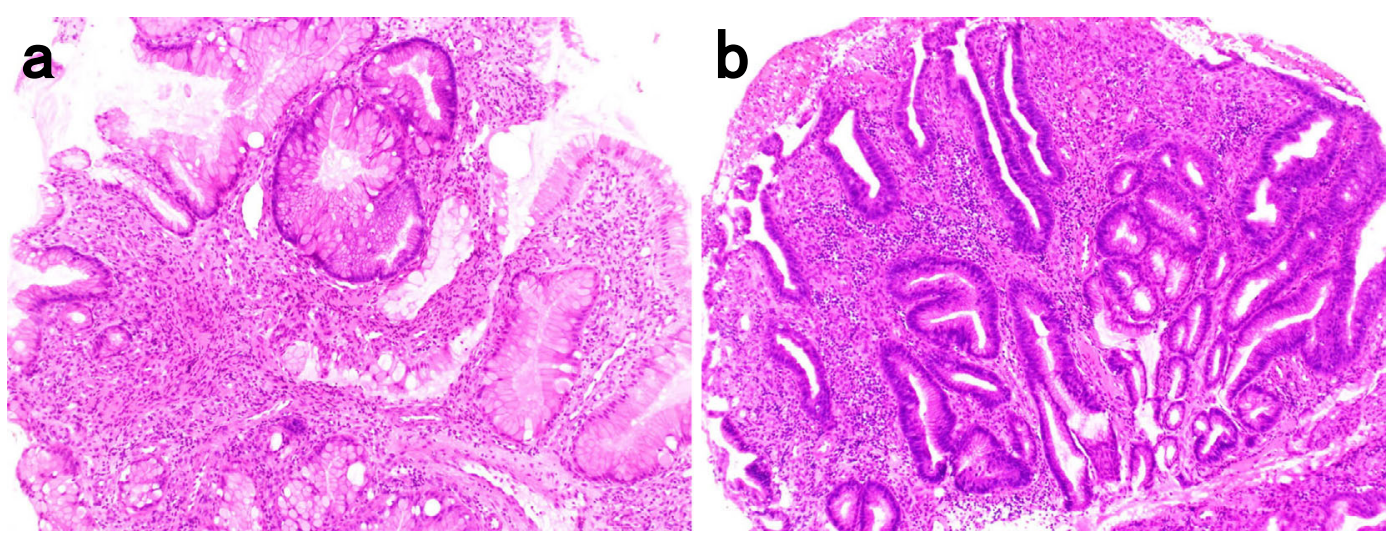

Figure 4. Biopsy specimens from gastric polyps of the antrum (a) and angle (b) indicated hyperplasia of the foveolar epithelium with edema and capillary dilation (Hematoxylin and Eosin staining, $\times 100)$.


Figure 5. One year after the fourth endoscopy, a fifth endoscopy revealed that all polyps and the remaining GAVE had completely disappeared (a-d). GAVE: gastric antral vascular ectasia

(Fig. 6).

\section{Discussion}

Hyperplastic gastric polyps developing after electrocoagulation therapy for GAVE were first reported after endoscopic laser therapy by Geller et al. in 1996 (14). In 1998, Dohmen et al. reported the first Japanese case of gastric hyperplastic polyps at four months after heater probe therapy in a patient with liver cirrhosis (15). Subsequent reports described the development of gastric polyps as a complication of endoscopic therapy, especially APC, for the treatment of
GAVE (16-20). This is the first reported case whereby switching from a PPI to an $\mathrm{H} 2$ blocker antagonist led to the disappearance of gastric polyps that appeared after endoscopic therapy for GAVE.

The histological findings of gastric polyps after the endoscopic treatment of GAVE indicated hyperplastic foveolar epithelium with the dilation and increase of capillaries, similar to common gastric hyperplastic polyps (14-20). The gastric polyps in our case showed a similar histology. Previous studies of gastric polyps in patients with portal hypertension used the terms "portal hypertensive polyp," "gastric polyps in patients with portal hypertension," or "portal 




Figure 6. Four and five years after APC therapy, the sixth (a, b) and seventh (c, d) endoscopic examinations, respectively, indicated the polyps and GAVE had not reappeared. Esophageal varix was not markedly different from that at the initial endoscopy (a). APC: argon plasma coagulation, GAVE: gastric antral vascular ectasia

hypertension-associated gastric polyp" (21-24).

The pathogenic mechanism of gastric hyperplastic polyps in patients with liver cirrhosis or portal hypertension is currently unclear, but several previous studies have suggested that congestion caused by elevated portal pressure might have an important role in inducing mucosal proliferation and angiogenesis (14-20). Furthermore, mucosal and vascular structural damage induced by APC might be involved in the pathogenesis rather than the superficial inflammation of the mucosa. In general, common gastric hyperplastic polyps arise from atrophic gastric mucosa caused by $H$. pylori infection (25-27) or autoimmune gastritis $(27,30,31)$. In our case, $H$. pylori infection was negative, and no atrophy of the gastric mucosa evaluated by endoscopy was found. Moreover, previous studies of the pathogenesis of gastric hyperplastic polyps have shown that hypergastrinemia induced by severe atrophic gastritis of the corpus (25-29) or prolonged use of PPIs might induce the development of gastric hyperplastic polys $(28,32,33)$. Gastrin has trophic effects on the gastrointestinal mucosa (34) and may repair gastric mucosal damage caused by APC. When gastric hyperplastic polyps were diagnosed in the present case, hypergastrinemia (817 $\mathrm{pg} / \mathrm{mL}$ ) caused by PPIs was found, and the gastrin level was normalized $(121 \mathrm{pg} / \mathrm{mL})$ by changing the treatment to an $\mathrm{H} 2$ blocker antagonist and rebamipide, with all gastric polyps and iron deficiency anemia completely disappearing. Rebamipide was used because it decreases gastrin levels in the blood and repairs damaged gastric mucosal tissues (35-37). PPIs were reported to be related to iron deficiency anemia $(38,39)$; therefore, the discontinuation of PPIs might have been involved in the improvement of anemia in this case.

Recently, Okazaki et al. reported gastric hyperplastic polyps in a patient with gastroesophageal reflux disease, which might have been caused by the prolonged use of PPIs, disappeared one year after switching from a PPI to an $\mathrm{H} 2$ receptor antagonist (40). PPIs were suspected to have caused the development of gastric hyperplastic polyps because $H$. pylori infection was negative and atrophic gastritis was not found. In general, common gastric hyperplastic polyps develop from atrophic gastritis induced by $H$. pylori infection (25-27) or autoimmune gastritis with hypergastrinemia $(30,31)$. Unfortunately, the level of serum gastrin was not described in that case study. Anjiki et al. reported a case of multiple hyperplastic polyps with adenocarcinoma in which hypergastrinemia was induced by the long-term use 
of PPIs; however, the gastric polyps disappeared and gastrin levels normalized after the discontinuation of PPIs (41). That case was $H$. pylori-positive, and $H$. pylori eradication therapy was performed in addition to the discontinuation of PPIs. H. pylori eradication therapy was reported to normalize serum gastrin levels $(42,43)$ and reduce gastric hyperplastic polyps $(43,44)$. In our case, which was negative for H. pylori and atrophic gastritis, the gastric hyperplastic polyps disappeared completely with the normalization of gastrin levels.

Our patient had liver cirrhosis with hepatocellular carcinoma, and various factors might have been involved in the disappearance of the polyps over a long period; however, such polyps do not disappear spontaneously. PPIs are useful for treating reflux esophagitis and peptic ulcer diseases as well as for $H$. pylori eradication therapy and the prevention of peptic ulcer diseases. Furthermore, they are often used long-term in general practice. When hyperplastic polyps are diagnosed after APC treatment of GAVE, hypergastrinemia induced by PPIs should be considered, as in the present case, and treatment by decreasing the PPI dose or switching from a PPI to an $\mathrm{H} 2$ receptor antagonist with rebamipide might be suitable.

\section{Informed consent was obtained from the patient.}

The authors state that they have no Conflict of Interest (COI).

\section{Acknowledgement}

We thank J. Ludovic Croxford, PhD for editing a draft of this manuscript.

\section{References}

1. Toyonaga A, Iwao T. Portal-hypertensive gastropathy. J Gastroenterol Hepatol 13: 865-877, 1998.

2. Burak KW, Lee SS, Beck PL. Portal hypertensive gastropathy and gastric antral vascular ectasia (GAVE) syndrome. Gut 49: 866-872, 2001.

3. Merli M, Nicolini G, Angeloni S, et al. The natural history of portal hypertensive gastropathy in patients with liver cirrhosis and mild portal hypertension. Am J Gastroenterol 99: 1959-1965, 2004.

4. Fontana RJ, Sanyal AJ, Ghany MG, et al. Development and progression of portal hypertensive gastropathy in patients with chronic hepatitis C. Am J Gastroenterol 106: 884-893, 2011.

5. Patwardhan VR, Cardenal A. Review article: the management of portal hypertensive gastropathy and gastric antral ectasia in cirrhosis. Aliment Pharmacol Ther 40: 354-362, 2014.

6. Gjeorgjievski M, Cappell MS. Portal hypertensive gastropathy: a systematic review of the pathophysiology, clinical presentation, natural history and therapy. World J Hepatol 8: 231-262, 2016.

7. Rider JA, Klotz AP, Kirsner JB. Gastritis with veno capillary ectasia as a source of massive gastric hemorrhage. Gastroenterology 24: 118-123, 1953.

8. Herrera S, Bordas JN, Llach J, et al. The beneficial effects of argon plasma coagulation in the management of different types of gastric vascular ectasia lesions in patients admitted for GI hemorrhage. Gastrointest Endosc 68: 440-446, 2008.

9. Fuccio L, Zagari RM, Serrani M, et al. Endoscopic argon plasma coagulation for the treatment of gastric antral vascular ectasia -
Related bleeding in patients with liver cirrhosis. Digestion 79: 143-150, 2009

10. Chiu YC, Lu LS, Wu KL, et al. Comparison of argon plasma coagulation in management of upper gastrointestinal angiodysplasia and gastric antral vascular ectasia hemorrhage. BMC Gastroenterol 12: $67,2012$.

11. Dulai GS, Jensen DM, Kovacs TOG, et al. Endoscopic treatment outcomes in watermelon stomach patients with and without portal hypertension. Endoscopy 36: 68-72, 2004.

12. Sato T, Yamazaki K, Toyota J, et al. Efficacy of argon plasma coagulation for gastric antral vascular ectasia associated with chronic liver disease. Hepatol Res 32: 121-126, 2005.

13. Kwan V, Bourke MJ, Williamas SJ, et al. Argon plasma coagulation in the management of symptomatic gastrointestinal vascular lesions: experience in 100 consecutive patients with long-term follow-up. Am J Gastroenterol 101: 58-63, 2006.

14. Geller A, Gostout CJ, Balm RK. Development of hyperplastic polyps following laser therapy for watermelon stomach. Gastrointest Endosc 43: 54-56, 1996.

15. Dohmen K, Ohtsuka Y, Takahashi K, et al. A case of multiple hyperplastic polyps arosen from scars caused by heater probe treatment for gastric antral vascular ectasia. Gastrointest Endosc 40: 901-906, 1998 (in Japanese, Abstract in English).

16. Izquierdo S, Rey E, del Olmo G, et al. Polyps as a complication of argon plasma coagulation in watermelon stomach. Endoscopy 37: 921, 2005.

17. Farooq FT, Wong RCK, Yang P, et al. Gastric outlet obstruction as a complication of argon plasma coagulation for watermelon stomach. Gastrointest Endosc 65: 1090-1092, 2007.

18. Ghabril M, Gross S, Krishna M, et al. Hyperplastic polyposis following treatment of gastric vascular ectasia: a case report and review of clinical correlates. Case Rep Gastroenterol 1: 48-52, 2007.

19. Shah N, Cavanagh Y, Kaswala DH, et al. Development of hyperplastic polyps following argon plasma coagulation of gastric antral vascular ectasia. J Nat Sci Biol Med 6: 479-482, 2015.

20. Kawaguchi K, Haratake J. Multiple hyperplastic polyps following argon plasma coagulation of gastric antral vascular ectasia. Gastroenterol Endosc 57: 52-53, 2015 (in Japanese).

21. Lam MCW, Tha S, Owen D, et al. Gastric polyps in patients with portal hypertension. Eur J Gastroenterol Hepatol 23: 1245-1249, 2011.

22. Amarapurkar AD, Amarapurkar D, Choksi M, et al. Portal hypertensive polyps: distinct entity. Indian J Gastroenterol 32: 195-199, 2013.

23. Livovsky DM, Pappo O, Skarzhinsky G, et al. Gastric polyp growth during endoscopic surveillance for esophageal varices or Barrett's esophagus. Isr Med Assoc J 18: 267-271, 2016.

24. Kara D, Husing-Kabar A, Schmidt H, et al. Portal hypertensive polyposis in advanced liver cirrhosis: the unknown entity? Can J Gastroenterol Hepatol 2018: 2182784, 2018.

25. Jain R, Chetty R. Gastric hyperplastic polyps: a review. Dig Dis Sci 54: 1839-1846, 2009.

26. Abraham Sc, Singh VK, Yardley JH, et al. Hyperplastic polyps of the stomach. Associations with histologic patterns of gastritis and gastric atrophy. Am J Surg Pathol 25: 500-507, 2001.

27. Haruma K, Yoshihara M, Sumii K, et al. Gastric acid secretion, serum pepsinogen I, and serum gastrin in Japanese with gastric hyperplastic polyps or polypoid-type early gastric carcinoma. Scand J Gastroenterol 28: 633-637, 1993.

28. Hongo M, Fujimoto K; Gastric Polyps Study Group. Incidence and risk factor of fundic gland polyp and hyperplastic polyp in long-term proton pump inhibitor therapy: a prospective study in Japan. J Gastroenterol 45: 618-624, 2010.

29. Hu H, Zhang Q, Chen G, et al. Risk factors and clinical correlates of neoplastic transformation in gastric hyperplastic polyps in Chinese patients. Sci Rep 10: 2582, 2020. 
30. Stockbrugger RW, Menon GG, Beilby JOW, et al. Gastroscopic screening in 80 patients with pernicious anaemia. Gut 24: 11411147, 1983.

31. Terao S, Suzuki S, Yaita H, et al. Multicenter study of autoimmune gastritis in Japan: clinical and endoscopic characteristics. Dig Endosc 32: 364-372, 2020.

32. Choudhry U, Boyce HW, Coppola D. Proton pump inhibitorassociated gastric polyps. A retrospective analysis of their frequency, and endoscopic, histologic, and ultrastructural characteristics. Am J Clin Pathol 110: 615-621, 1998.

33. Miyamoto S, Kato m, Matsuda K, et al. Gastric hyperplastic polyps associated with proton pump inhibitor use in a case without a history of Helicobacter pylori infection. Intern Med 56: 18251829, 2017.

34. Haruma k, Kamada T, Manabe N, et al. Old and new gut hormone, gastrin and acid suppressive therapy. Digestion 97: 340$344,2018$.

35. Haruma K, Ito M, Kido S, et al. Long-term rebamipide therapy improves Helicobacter pylori-associated chronic gastritis. Dig Dis Sci 47: 862-867, 2002.

36. Kinjo N, Kawanaka H, Akshodhi T, et al. Significance of ERK nitration in portal hypertensive gastropathy and its therapeutic implications. Am J Physiol Gastrointest Liver Physiol 295: G1016G1024, 2008.

37. Haruma K, Ito M. Review article: clinical significance of mucosalprotective agents: acid, inflammation, carcinogenesis and rebamipide. Aliment Pharmacol Ther 18: 153-159, 2003.
38. Sarzynski E, Putarajappa C, Xie Y, et al. Association between proton pump inhibitor use and anemia: a retrospective cohort study. Dig Dis Sci 56: 2349-2353, 2011.

39. Lam JR, Schneider JL, Quesenberry CP, et al. Proton pump inhibitor and histamine-2 receptor antagonist use and iron deficiency. Gastroenterology 152: 821-829, 2017.

40. Okazaki Y, Kotani K, Higashi Y. Vanishing gastric hyperplastic polyps. BMJ Case Rep 12: e231341, 2019.

41. Anjiki H, Mukaisho K, Kadomoto Y, et al. Adenocarcinoma arising in multiple hyperplastic polyps in a patient with Helicobacter pylori infection and hypergastrinemia during long-term proton pump inhibitor. Clin J Gastroenterol 10: 128-136, 2017.

42. Wagner S, Haruma K, Gladziwa U, et al. Helicobacter pylori infection and serum pepsinogen A, pepsinogen $\mathrm{C}$, and gastrin in gastritis and peptic ulcer: significance of inflammation and effect of bacterial eradication. Am J Gastroenterol 89: 1211-1218, 1994.

43. Ohkusa T, Takashimizu I, Fujiki K, et al. Disappearance of hyperplastic polyps in the stomach after eradication of Helicobacter pylori. A randomized, controlled trial. Ann Intern Med 129: 712-715, 1998.

44. Ji F, Wang ZW, Ning JW, et al. Effect of drug treatment on hyperplastic gastric polyps infected with Helicobacter pylori: a randomized, controlled trial. World J Gastroenterol 12: 1770-1773, 2006.

The Internal Medicine is an Open Access journal distributed under the Creative Commons Attribution-NonCommercial-NoDerivatives 4.0 International License. To view the details of this license, please visit (https://creativecommons.org/licenses/ by-nc-nd/4.0/).

(C) 2021 The Japanese Society of Internal Medicine Intern Med 60: 1019-1025, 2021 